\title{
Efeito de óleos essenciais e composto citronelal sobre a micoflora de sementes de feijão armazenadas
}

\section{Effect of essential oils and citronellal compound on bean seeds stored microflora}

\author{
Marcio Akio Ootani ${ }^{1}$; Deyvid Rocha Brito ${ }^{2}$; Gabriela Priscila de Sousa Maciel ${ }^{3}$; Larissa Alves Lopes ${ }^{4}$; \\ Raimundo Waguer de Souza Aguiar ${ }^{5}$
}

\begin{abstract}
Resumo: Esse trabalho objetivou avaliar o efeito de óleos essenciais de Cymbopogon nardus, Eucalyptus citriodora e o composto citronelal como meios alternativos na conservação e qualidade sanitária de sementes de diferentes genótipos de feijão. Utilizou-se o delineamento inteiramente casualizado, com 200 sementes subdivididas em repetições de 50 para cada tratamento. As sementes de feijão Carioca, Caupi, Jalo e Preto, foram tratados com os referidos óleos essenciais nas doses de 5, 10, 20, 30\% e Testemunha (tratadas apenas com água destilada esteril), posteriormente foram armazenadas por 15, 30, 45 e 60 dias. No final de cada período de armazenamento, foram realizados os bioensaios utilizando o método de Blotter test, para avaliação e identificação das colônias de fungos associados as sementes. os dados foram submetidos a análise de variância e as médias comparadas pelo teste de Tukey a 5\% de probabilidade Os óleos utilizados se mostraram eficientes, por terem promovido o decréscimo no número de colônias em todas as doses para as sementes dos quatro genótipos de feijão ao longo do armazenamento, com destaque para as maiores doses. Analisando as sementes testemunhas, sendo os fungos Aspergillus sp.,Fusarium sp. e Penicillium sp. que apresentaram o maior número de colônias, respectivamente. Dentre os óleos utilizados, o composto citronelal e o oleo de citronela teve efeito na inibição dos fungos associados às sementes em todos os genótipos utilizados, em todas as doses.
\end{abstract}

Palavras - chave: Controle alternativo, fungos, diversidade e qualidade fitossanitária.

\begin{abstract}
This study aimed to evaluate the effect of essential oils of Cymbopogon Nardus, Eucalyptus citriodora and citronellal compound as alternative means conservation and sanitary quality of seeds of different genotypes. We used a completely randomized design, with 200 seeds subdivided into 50 repetitions for each treatment. The bean seeds Carioca, Caupi, Jalo and Preto, were treated with these essential oils in doses of 5, 10, 20, 30\% and control (treated only with distilled water and autoclaved) were subsequently stored for 15, 30, 45 and 60 days. At the end of each storage period, the bioassays were performed using the Blotter test method for evaluation and identification of fungi colonies associated with the seed. the data were subjected to analysis of variance and the means compared by Tukey test at 5\% probability. The oils used were efficient because they promoted the decrease in the number of colonies at all doses for the seeds of the four genotypes during storage, especially for larger doses. Analyzing the seeds witnesses, and the Aspergillus sp., Fusarium sp. and Penicillium sp. which had the largest number of colonies, respectively. Among the oils used, citronellal compound and C. nardus oil had no effect on inhibition of fungi in the seeds in all genotypes and in all doses.
\end{abstract}

Keywords: Alternative control, fungi diversity and plant quality.

\footnotetext{
*Autor para correspondência

Recebido para publicação em 29/11/2015; aprovado em 12/03/2016

${ }^{1}$ Doutorando em Fitotecnia. Universidade Federal do Ceara / Departamento de Fitotecnia/CCA/UFC; Fortaleza - Ceara; (85) 33669670. E-mail: ootani667@uft.edu.br

${ }^{2}$ Mestre em Produção Vegetal. Universidade Federal do Ceara / Departamento de Ciências Agrária; Gurupi - Tocantins. E-mail: dvbrito06@ hotmail.com ${ }^{3}$ Graduanda em Agronomia. Universidade Federal do Ceara / Departamento de Fitotecnia/CCA/UFC; Fortaleza - Ceara. E-mail: macielpriscilagabi20@hotmail.com

${ }^{4}$ Graduanda e Biotecnologia; Universidade Federal do Ceara / Departamento de Bioquimica; Fortaleza - Ceara. E-mail: larissalveslopes@gmail.com

${ }^{5}$ Professor Adjunto; Universidade Federal do Ceara Departamento de Ciências Agrária; Gurupi - Tocantins. E-mail: rwsa@mail.uft.edu.br
} 


\section{INTRODUÇÃO}

O feijão-comum (Phaseolus vugalis L.) é uma oleaginosa importante na dieta da população brasileira, por ser uma excelente fonte proteica, além de possuir entre seus constituintes os carboidratos e ferro (CÂMARA;URREA, 2013). Outra espécie muito cultivada também é o feijão caupi (Vigna unguiculata L.) Conhecido como feijão-decorda. Por questões abióticas como alta temperatura e alta umidade, esse feijão adaptou-se as regiões norte e nordeste do Brasil (HARUNA;USMAN, 2013). O feijão é um dos alimentos mais tradicionais na culinária brasileira, com significativa importância social e econômica (STONE et al., 2013).

No entanto, como na maioria das espécies cultivadas, os agentes etiológicos das doenças do feijão caupi e comum, são transportados pelas sementes, principalmente os fungos que reduzem o poder germinativo e podem ser disseminados, estabelecendo assim focos primários de infecção em novas áreas de cultivo (MARINO et al., 2008). Do ponto de vista fitopatológico, a importância da associação patógeno-semente não reside apenas no fato de ser mais um processo de disseminação, mas também por se constituir num meio de sobrevivência do patógeno em contato direto com o hospedeiro (REY et al., 2009). Isso implica em consequências diretas, tais como dispersão de raças mais agressivas, ainda não existentes na área, e assegura a introdução do patógeno já nos primeiros estágios de desenvolvimento da planta. Além disso, a incidência de doenças já existentes numa área tende a aumentar com a frequente introdução de patógenos via semente (RANI et al., 2013).

O manejo integrado de doenças envolve todas as estratégias disponíveis para manter as populações dos patógenos abaixo do limiar de dano econômico e ao mesmo tempo minimizar os efeitos negativos no ambiente. Isso não deve ser visto como uma simples sobreposição de duas ou mais técnicas de controle. Como comentam Montemor et al. (2011), a sanidade de sementes tem sido característica relevante no desempenho das sementes. A má qualidade sanitária das sementes contribui para a sua baixa germinação, redução do número de plantas no estande, podridões radiculares, murchas e consequentemente, baixo rendimento da cultura. É grande a diversidade fúngica encontrada em sementes de Feijão, principalmente dos gêneros Penicillium sp, Aspergillus sp. e Curvularia sp. (RODRIGUES; MENEZES, 2002).

Devido a procura por alternativas eficientes no controle desses fitopatógenos, sem implicações adversas, aliada ao crescimento da demanda de consumo por produtos alimentares seguros, vem se intensificando nos últimos anos a procura por métodos de controle alternativos (ZOTTICH et al., 2013). Dentre eles, destaca os metabolitos secundários, com propriedades antifúngicas e antimicrobianas (SANTOS et al., 2007; AHMADI et al., 2010). Existem relatos de que os óleos essenciais de Eucaliptus sp. e Cymbopogon nardus possuem constituintes que podem apresentar atividade fungitóxica, reduzindo o crescimento micelial e a produção de esporos e germinação de vários fungos de alguns ambientes (VILELA et al., 2009). Esses óleos essenciais podem apresentar atividade direta ou indireta pelos metabólitos secundários, que são encontrados pela sintetização destes pela planta para a defesa da mesma contra patógenos (SCHWAN-ESTRADA e STANGARLIN, 2005; SU et al., 2006).

Levando em consideração o contexto levantado e a importância desses fungos no agroecossistema e ambiente de armazenamento de sementes, objetivou-se avaliar o efeito dos óleos essenciais de Cymbopogon nardus, Eucalyptus cytriodora e composto citronelal sobre a micoflora associada a sementes de feijão comum, Caupi, Jalo e Preto em função de de ferentes concentração de óleos essenciais e períodos de armazenamento das sementes.

\section{MATERIAL E MÉTODOS}

O trabalho foi realizado no laboratório de Biotecnologia, localizado na Universidade Federal do Tocantins, campus de Gurupi, no período de fevereiro a setembro de 2013. O delineamento experimental utilizado foi o inteiramente casualizado, sendo quatro genótipos de feijão (carioca, preto, jalo e caupi), três tipos de óleos essenciais (Cymbopogon nardus, Eucalyptus cytriodora e composto Citronelal), quatro períodos de armazenamento $(15,30,45$ e 60 dias), quatro concentrações dos referidos óleos $(5 ; 10 ; 20$ e $30 \%)$ e respectivamente, com 4 repetições para cada tratamento.

Para a obtenção dos óleos para os testes, foi seguida a metodologia descrita por Castro et al. (2006). As plantas foram colhidas no Campus Universitário de Gurupi na horta de plantas medicinas, onde as folhas de E. citriodora e $C$. nardus foram picadas e pesados $300 \mathrm{~g}$ e adicionado $500 \mathrm{ml}$ de água por extração. Em seguida foram colocadas no balão de $1000 \mathrm{ml}$. A obtenção dos óleos foi por hidrodestilador modelo Clevenger (arraste de vapor) e deixado por um período de 2,5 horas. Coletou-se o hidrolato, que em seguida foi centrifugado a 1000 RPM por 5 minutos. O óleo foi retirado com o auxílio de uma micropipeta e posteriormente foi acondicionado em frasco de vidro âmbar envolto com papel alumínio. Após, colocou-se sob refrigeração a $4^{\circ} \mathrm{C}$ para evitar sua volatilização e fotooxidação devido aos compostos terpenoides. A obtenção do composto citronelal que é um composto majoritário presente no óleo essencial do Eucaliptus citriodora foi obtida junto à empresa Sigma-Aldrich com grau de pureza de $85 \%$, em frasco de $100 \mathrm{~mL}$.

A análise dos óleos foi feita por CG/EM (cromatografo gasoso/espectrômetro de massa) utilizando aparelho Shimadzu QP5050 quadrupole com a coluna de capilaridade com sílica $(30 \mathrm{~m}$ x 0,25 $\mathrm{mm}$ x 0,25 $\mu \mathrm{m})$. Programou-se a temperatura da coluna para $40^{\circ} \mathrm{C}$ por $2 \mathrm{~min}$, variando de $220^{\circ} \mathrm{C}$ min $^{-1}$ para $280^{\circ} \mathrm{C}$ à $20^{\circ} \mathrm{C}$ min $^{-1}$ para integração. As temperaturas do injetor e detector foram de $250^{\circ} \mathrm{C}$ e $280^{\circ} \mathrm{C}$, respectivamente. Usou-se o hélio como carreador gasoso e fluxo de $1,5 \mathrm{~mL} \min ^{-1}$ (1:50). Aplicou-se $1 \mu \mathrm{L}$ de $1 / 100$ de solução diluída em acetato de etila. $\mathrm{O}$ espectro de massa foi obtido à $70 \mathrm{eV}$ e a velocidade de leitura foi $0,5 \mathrm{scan} \mathrm{s}^{-1}$ de 40 à $650 \mathrm{~m} / \mathrm{z}$. A identificação dos compostos majoritários foi realizada com base na comparação dos índices de retenção (VANDENDOLL;KRATZ, 1963) bem como por comparação computadorizada do espectro de massa obtido com aqueles contidos na biblioteca de espectro de massas do NIST do banco de dados GC/EM (SHELLIE et al., 2002). Os compostos presentes nos óleos essenciais de $E$. citriodora e $C$. nardus constam na Tabela 1. 
No experimento foi avaliado o efeito desses óleos sobre sementes de quatro genótipos de feijão, sendo Carioca, Caupi, Jalo e Preto, tratando-os com os referidos óleos essenciais (Cymbopogon nardus, Eucalyptus cytriodora e composto Citronelal) nas concentrações de 5, 10,20 e $30 \%$. No experimento ainda continha as sementes tratadas com água destilada e autoclavada (testemunha). Para cada tratamento estabelecido, foram selecionadas 200 sementes divididas em 50 sementes por repetição, em seguida foram armazenadas por 15, 30, 45 e 60 dias em potes de $200 \mathrm{~mL}$ para cada óleo essencial e suas respectivas concentrações as concentrações em função dos óleos e do composto majoritário os dados submetidos a analise de regressão.

No final de cada período de armazenamento foram realizados os bioensaios pelo método Blotter test, descrito por Luca-Filho (1987), onde as sementes foram retiradas e acondicionas em placas de Petri de $20 \mathrm{~cm}$ de diâmetro forradas com papel filtro e umedecida com $15 \mathrm{ml}$ de água destilada estéril, posteriormente foram colocadas em câmara BOD à temperatura de $27^{\circ} \mathrm{C}$ visando o aparecimento das colônias. Após o período de incubação, avaliou-se a incidência dos fungos nas sementes com auxílio de um microscópio estereoscópico e microscópio composto, sendo os resultados expressos em números de sementes contaminadas com cada tipo de fungo. Os dados foram submetidos a analise fatorial (espécie de feijão $\mathrm{x}$ gênero dos fungos) e (óleos $x$ gênero de fungos) $\mathrm{e}$ as médias comparadas pelo teste de Tukey a $5 \%$ de probabilidade com auxílio do programa estatístico Sisvar 5.0 (FERREIRA, 2003).

\section{RESULTADOS E DISCUSSÃO}

Os óleos essenciais apresentaram sete constituintes para E. citriodora e dez para C. nardus que foram identificados por CG-EM. Entre os compostos majoritários encontrados no óleo de E. citriodora, destaca-se o citronelal com $61,78 \%$, seguido de isopolegol com $11,89 \%$. Enquanto, que no óleo de $C$. nardus os constituintes predominantes foram citronelal com $36,53 \%$, geraniol com $25,56 \%$ e $\beta$-citronelol 13,10 \%. Em todos os óleos analisados houve predominância dos monoterpenos conforme mostrados na Tabela 1.

Tabela 1. Compostos majoritários identificados de óleos essenciais de Eucalyptus citriodora e Cymbopogon nardus.

\begin{tabular}{lcc}
\hline Compostos & \multicolumn{2}{c}{ Composição dos óleos essenciais em \% } \\
& C. nardus & E. citriodora \\
\hline$\beta$-pineno & - & 2,83 \\
1,8-Cineol & - & 3,44 \\
Isopulegol & 1,40 & 15,54 \\
Citronelal & 36,53 & 61,78 \\
Citronelol & 13,10 & 7,90 \\
Carriofileno & - & 2,13 \\
Geraniol & 25,56 & - \\
Acetato de citronelil & 2,22 & - \\
Acetato de geranil & 1,51 & - \\
Germacreno-D & 0,69 & - \\
Delta cadileno & 1,09 & - \\
Elemol & 8,24 & - \\
Germecreno-D-4-ol & 1,64 & - \\
\hline Total & 91,98 & 93,62 \\
\hline
\end{tabular}

Estes óleos essenciais são citados como possuidor de mais de 80 substâncias, a partir do qual citronelal, citronelol, geraniol, limoneno e ésteres têm particular importância (DAVID et al., 2007; MARCO et al., 2007). Estes resultados confirmam os encontrados por outros pesquisadores em relatos anteriores, onde é registrado que citronelal e geraniol são os principais constituintes do óleo essencial de C. nardus e até mesmo de outras plantas (SIMIC et al., 2008).

Tabela 2. Análise de variância da interação entre cultivares e fungos de sementes de feijão ao final de 60 dias de armazenamento.

\begin{tabular}{lcccccc}
\hline Cultivares & Aspergillus $\mathrm{sp}$. & Curvularia $\mathrm{sp}$. & Fusarium $\mathrm{sp}$. & Nigrospora $\mathrm{sp}$. & Penicilium $\mathrm{sp}$. & Médias \\
\hline Carioca & $11,0791 \mathrm{Ba}$ & $0,4833 \mathrm{Bc}$ & $1,7541 \mathrm{Cc}$ & $0,3666 \mathrm{ABc}$ & $4,8500 \mathrm{Bb}$ & $3,7066 \mathrm{C}$ \\
Caupi & $3,7166 \mathrm{Cb}$ & $0,0000 \mathrm{Bc}$ & $18,9333 \mathrm{Aa}$ & $0,2000 \mathrm{Bc}$ & $0,5666 \mathrm{Cc}$ & $4,6841 \mathrm{~B}$ \\
Jalo & $18,4875 \mathrm{Aa}$ & $0,2333 \mathrm{Bc}$ & $1,0500 \mathrm{Cc}$ & $0,0000 \mathrm{Bc}$ & $6,3333 \mathrm{Ab}$ & $5,2208 \mathrm{~B}$ \\
Preto & $19,6333 \mathrm{Aa}$ & $2,0333 \mathrm{Acd}$ & $3,4583 \mathrm{Bc}$ & $1,6500 \mathrm{Ad}$ & $7,3291 \mathrm{Ab}$ & $6,8208 \mathrm{~A}$ \\
\hline \multicolumn{1}{c}{ Médias } & $13,2291 \mathrm{a}$ & $0,6875 \mathrm{~d}$ & $6,3000 \mathrm{~b}$ & $0,5541 \mathrm{~d}$ & $4,7697 \mathrm{c}$ \\
\multicolumn{1}{c}{ CV $(\%)$} & 26,12 & & & & & \\
\hline
\end{tabular}

Letras seguidas de mesma letra maiúscula na coluna e minúscula na linha, não diferem significativamente entre sí pelo teste de Tukey a $5 \%$ de probabilidade.
$\mathrm{Na}$ avaliação da micoflora após os tratamentos, no geral, observou-se o aparecimento de vários gêneros de fungos, entre eles o Aspergillus sp. que teve incidência de 7,42 a $40 \%$, Fusarium sp. de 2 a $38 \%$, Penicilium sp. de 1 a $14 \%$ e os demais fungos não ultrapassaram $4 \%$, sendo que essas variações dependeram da espécie de feijão, tipo de óleo e suas concentrações, conforme mostrado nas tabelas 2 e 3 . 
Segundo resultados encontrados por Torres et al. (1997), apesar da constante presença de Aspergillus niger, Aspergillus flavus, Trichoderma sp. e Penicillium digitatum, com frequências de 51,12, 2 e $1 \%$, respectivamente, os lotes de grãos analisados não mostraram seu vigor afetado. Do ponto de vista fitopatológico, a importância da associação patógeno-semente não reside apenas no fato de ser mais um processo de disseminação, mas também por se constituir num meio de sobrevivência do patógeno em contato direto com o hospedeiro. Isto implica em consequências diretas, tais como a introdução de patógenos em áreas ainda não infestadas e de novas raças mais virulentas, ainda não existentes na área, e assegura a introdução do patógeno já nos primeiros estágios de desenvolvimento da planta. Além disso, a incidência de doenças já existentes numa área tende a aumentar com a frequente introdução de patógenos pela semente (GOMES et al., 2008), contudo o bioensaio demonstrou alta susceptibilidade de algumas cultivares como o feijão preto e o jalo para os fungos Aspergillus sp. e Penicilium sp. considerados contaminantes, por outro a lado o feijão Caupi teve maior incidência de Fusarium sp. A maioria dos agentes etiológicos das doenças do caupi é transmitida por sementes, principalmente as causadas por fungos que reduzem o poder germinativo e podem ser disseminados, estabelecendo assim focos primários de infecção em novas áreas de cultivo (MACHADO, 1994).

O efeito dos óleos essenciais de Cymbopogon nardus, Eucalyptus cytriodora e composto Citronelal sobre os fungos associados às sementes armazenadas promoveu inibição de todos os fungos Aspergillus sp., Curvularia sp., Nigrospora sp. e Penicilium sp. A ação de inibição dos óleos sobre os fungos está demonstrada na Tabela 3. Percebe-se que no geral, para as quatro cultivares de feijão, houve uma redução no número de colônias quando as sementes foram tratadas com os óleos essenciais de Cymbopogon nardus, Eucalyptus cytriodora e composto Citronelal em função da concentração, , com destaque para o óleo de citronela e o composto citronelal, que chegou a inibir $100 \%$ das colônias associadas às semente do feijão Caupi a partir da concentração de $10 \%$. Para os demais óleos (C.nardus e E. cytriodora) e o composto majoritário, verificou-se que houve diferença significativa entre as sementes tratadas com os óleos essenciais em todas as concentrações utilizadas. Contudo, fica claro o efeito fungitóxico dos produtos utilizados no presente estudo (Cymbopogon nardus, Eucalyptus cytriodora e composto Citronelal) sobre a microflora associada às sementes de feijão das quatro cultivares (preto, carioca, caupi e jalo).

Tabela 3- Analise de variância da interação entre os óleos essências (Cymbopogon nardus, Eucalyptus cytriodora e composto Citronelal) e fungos associados as sementes de feijão ao final de 60 dias de armazenamento.

\begin{tabular}{lcccccc}
\hline \multicolumn{1}{c}{ Óleos } & Aspergillus $\mathrm{sp}$. & Curvularia $\mathrm{sp}$. & Fusarium $\mathrm{sp}$. & Nigrospora $\mathrm{sp}$. & Penicillium $\mathrm{sp}$. & Médias \\
\hline C. $n$ ardus & $8,975 \mathrm{Ca}$ & $0,637 \mathrm{Ad}$ & $2,515 \mathrm{Cc}$ & $0,300 \mathrm{ABd}$ & $4,096 \mathrm{Bb}$ & $3,350 \mathrm{~B}$ \\
Citronelal & $14,134 \mathrm{Ba}$ & $0,887 \mathrm{Ad}$ & $9,950 \mathrm{Ab}$ & $0,125 \mathrm{Bd}$ & $5,237 \mathrm{Ac}$ & $6,066 \mathrm{~A}$ \\
E. citriodora & $16,578 \mathrm{Aa}$ & $0,537 \mathrm{Ad}$ & $6,434 \mathrm{Bb}$ & $1,237 \mathrm{Ad}$ & $4,975 \mathrm{ABc}$ & $5,952 \mathrm{~A}$ \\
\hline \multicolumn{1}{c}{ Médias } & $13,229 \mathrm{a}$ & $0,687 \mathrm{~d}$ & $6,300 \mathrm{~b}$ & $0,554 \mathrm{~d}$ & $4,769 \mathrm{c}$ & \\
\multicolumn{1}{c}{ CV $(\%)$} & 33,21 & & & & & \\
\hline
\end{tabular}

Letras seguidas de mesma letra maiúscula na coluna e minúscula na linha, não diferem significativamente entre sí pelo teste de Tukey a $5 \%$ de probabilidade.

Resultados semelhantes mostram efeito dos terpenoides sobre fungos fitopatogênicos Phytophora infestans (VELLUTI et al., 2004; PAWAR; THAKER, 2006; SOYLU et al., 2006), e na inibição micelial in vitro do fungo Monilinia fructicola, observou que o óleo essencial de $C$. nardus apresentou potencial para controle da doença nas concentrações de $20 \%$, superior ao óleo de $E$. citriodora (SOUZA et. al., 2005). Sobre os fungos C. musae e C. gloeosporioides, com inibição em $100 \%$ do crescimento micelial na concentração de $1000 \mu \mathrm{L} / \mathrm{L}$ a $1500 \mu \mathrm{L} / \mathrm{L}$ (MOLEYAR e NARASIMHAM, 2004).

$\mathrm{O}$ efeito dos óleos essenciais não variou o numero de colonias em função do período de armazenamento, permanecendo constante por um período de 60 dias após a aplicação, contudo variou em função concentração dos óleo essenciais e a espécie de feijão demonstrado na Tabela 4. Segundo Aguiar et al. (2014) testando in vitro óleos essenciais de E citriodora e C.nardus, no ensaio de contato inibiu do crescimento de três espécies de fungos, somente Aspergilus sp. na concentração de $1,47 \mathrm{mg} \mathrm{mL}^{-1}$ inibiu somente $90 \%$ em 14 dias. O efeito dos óleos e do composto citronelal foi maior no feijão carioca e caupi (Figura $1 \mathrm{~A} \mathrm{e}$ Figura $1 \mathrm{C}$ ) onde não se pode observar diferença entre os entre eles, contudo no feijão preto e jalo (Figura 1 B e Figura 1 D) pode observar que o óleo essencial de citronela e o composto citronelal foram os mais efetivos na inibição do numero de colônias fungicas associadas as sementes. Trabalhos de Khaledi et al. (2015) verificou a aplicação oleo essencial de hortelã-pimenta e mentol reduzido de forma significativa o desenvolvimento de doenças causadas por fungos de feijão Rhizoctonia solani e Macrophomina phaseolina. Observou-se maior capacidade óleo de menta e do hortelã na diminuição doenças de feijão.

Atividade antifúngica dos óleos essenciais depende do aumento da concentração aumenta a inibição do crescimento micelial fungos diferente. Efeito inibitório dos óleos essenciais no crescimento de fungos varia de acordo com a espécies dos fungos. Isto está de acordo com trabalhos anteriores sobre diferentes efeitos dos óleos essências sobre diferentes isolados de uma espécie fúngica (CHANG et al., 2008; AGUIAR et al., 2014).

A atividade dos óleos essenciais de C. nardus e $E$. citriodora contra fungos, pode estar ligado aos componentes majoritários como o citronelal, geraniol e citronelol, que pertencem a um dos grupos de constituintes comumente presentes em óleos essenciais (terpenos) que, de acordo com 
a literatura, agem principalmente contra a membrana citoplasmática dos microorganismos (DI PASQUA et al., 2007). Isto é justificado pelo caráter lipofílico destes compostos, sugerindo sua interação com membranas dos microrganismos. E de fato, a hidrofobicidade dessas moléculas as possibilita se particionarem nas membranas celulares dos fungos, alterando suas funções e as deixando mais permeáveis (SILVA; BASTOS, 2007). Sendo assim, o efeito como perturbação da membrana citoplasmática, ruptura do fluxo de elétrons, alteração no transporte de moléculas através da membrana, inibição de atividade de certas enzimas e coagulação do conteúdo citoplasmático são alguns mecanismos envolvidos na promoção do poder antimicrobiano dos óleos essenciais descritos na literatura (COX et al., 2000).

Em todas as amostras analisadas o fungo Penicillium sp. Ocorreu com incidência baixa e não foram verificadas diferenças estatisticamente entre as cultivares. Sallis et al. (2001) verificou a presença deste fungo em feijão-caupi no Rio Grande do Sul que é responsável pela deterioração de sementes durante a maturação e após a maturidade quando encontram condições de ambiente favoráveis ao seu desenvolvimento. Aspergillus sp. e Penicillium sp. são fungos de armazenamento comuns em sementes de feijãocaupi (NOVEMBRE e FILHO, 1991; GOMES et al., 2008).

Figura 1. Número médio de colônias de fungos associados a 50 sementes de feijão por repetição após tratamento com óleos essenciais de Cymbopogon nardus, Eucalyptus cytriodora e composto Citronelal após nas concentrações de 5, 10, 20 e 30\% dos oleos. Sendo: Figura 1 A = Sementes de feijão Carioca, Figura 1 B = Sementes de feijão Preto, Figura 1 C = Sementes de feijão Caupi e Figura 1 D = Sementes de feijão Jalo.
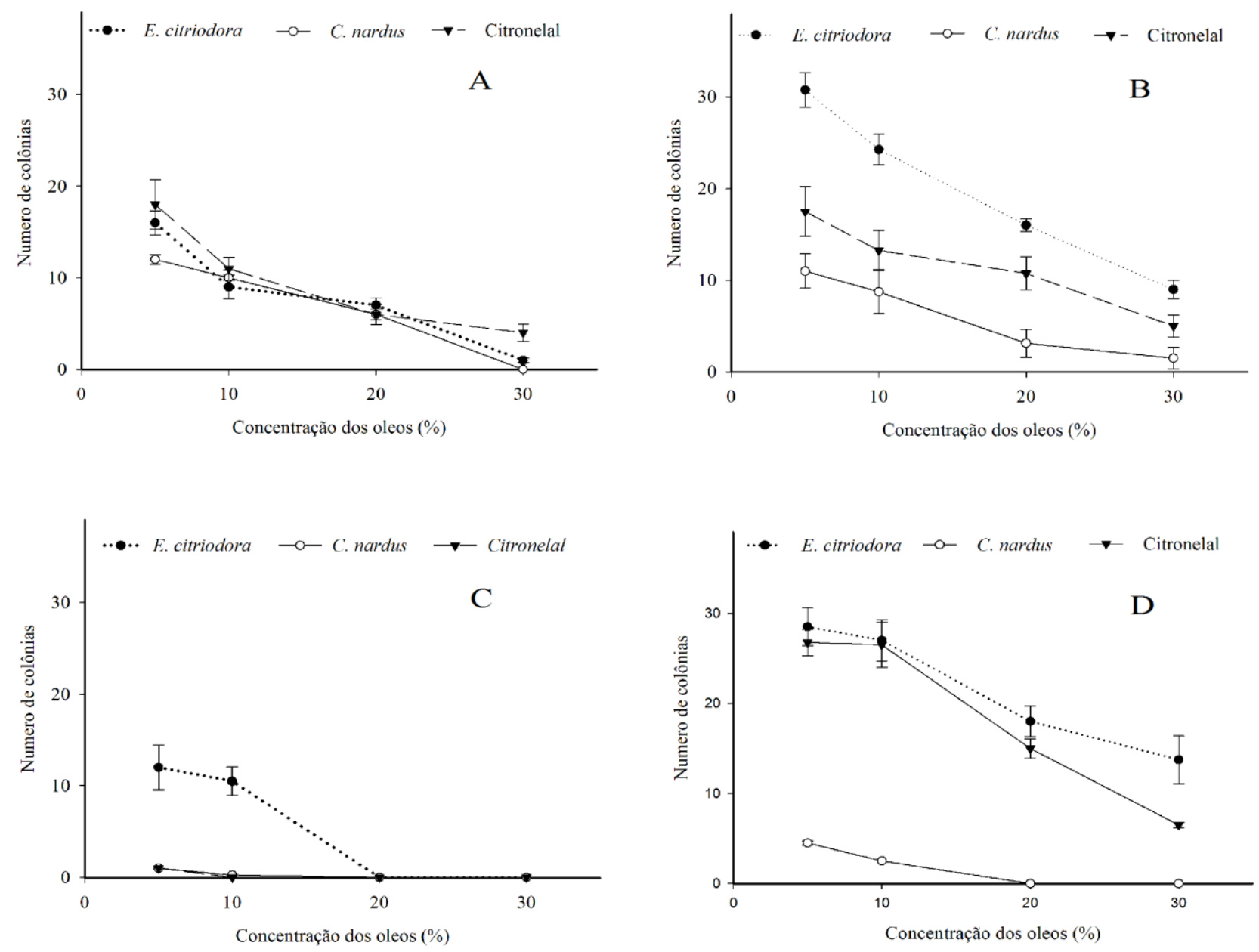

Em todas as amostras analisadas o fungo Penicillium sp. Ocorreu com incidência baixa e não foram verificadas diferenças estatisticamente entre as cultivares. Sallis et al. (2001) verificou a presença deste fungo em feijão-caupi no Rio Grande do Sul que é responsável pela deterioração de

sementes durante a maturação e após a maturidade quando encontram condições de ambiente favoráveis ao seu desenvolvimento. Aspergillus sp. e Penicillium sp. são fungos de armazenamento comuns em sementes de feijãocaupi (NOVEMBRE e FILHO, 1991; GOMES et al., 2008). 
Tabela 4. Análise de regressão do efeito das concentrações dos óleos essenciais e do composto majoritário em função da concentração sobre as sementes de feijão, a equação que melhor expressou o efeito dos óleos e do composto sobre o número de colônias foi $\mathrm{Y}=Y_{0}+a \mathrm{x}$.

\begin{tabular}{|c|c|c|c|c|c|}
\hline \multicolumn{6}{|c|}{ Carioca (A) } \\
\hline & $Y_{0}$ & $a$ & $\mathrm{~F}_{\text {regressão }}$ & $\mathrm{R}^{2}$ & $\mathrm{P}_{\text {regressão }}$ \\
\hline E. citriodora & $18,28 \mathrm{ab}$ & $-0,52 a$ & 13,61 & 0,80 & 0,0660 \\
\hline C. nardus & $14,71 b$ & $-0,47 \mathrm{a}$ & 175,00 & $0,98 * *$ & 0,0057 \\
\hline Citronelal & $16,89 \mathrm{a}$ & $-0,53 \mathrm{a}$ & 20,27 & $0,86^{*}$ & 0,0460 \\
\hline Media & 17,28 & $-0,53$ & & & \\
\hline \multicolumn{6}{|c|}{ Preto (B) } \\
\hline & $Y_{0}$ & $\mathrm{a}$ & $\mathrm{F}_{\text {regressão }}$ & $\mathrm{R}^{2}$ & $\mathrm{P}_{\text {regressão }}$ \\
\hline E. citriodora & $33,82 \mathrm{a}$ & $-0,85 b$ & 145,41 & $0,97 * *$ & 0,0060 \\
\hline C. nardus & $19,14 \mathrm{a}$ & $-0,46 a$ & 55,165 & $0,94 *$ & 0,0176 \\
\hline Citronelal & $12,53 b$ & $-0,39 a$ & 36,76 & $0,92 *$ & 0,0261 \\
\hline Media & 21,82 & $-0,56$ & & & \\
\hline \multicolumn{6}{|c|}{ Caupi (C) } \\
\hline & $Y_{0}$ & $\mathrm{a}$ & $\mathrm{F}_{\text {regressão }}$ & $\mathrm{R}^{2}$ & $\mathrm{P}_{\text {regressão }}$ \\
\hline E. citriodora & $14,46 a$ & $-0,54 b$ & 11,77 & 0,78 & 0,0700 \\
\hline C. nardus & $0,74 b$ & $-0,03 a$ & 1,68 & 0,18 & 0,3200 \\
\hline Citronelal & $1,90 \mathrm{~b}$ & $-0,08 \mathrm{a}$ & 2,35 & 0,31 & 0,2600 \\
\hline \multicolumn{6}{|l|}{ Media } \\
\hline \multicolumn{6}{|c|}{ Jalo (D) } \\
\hline & $\mathrm{Y}_{0}$ & $\mathrm{a}$ & $\mathrm{F}_{\text {regressão }}$ & $\mathrm{R}^{2}$ & $\mathrm{P}_{\text {regressão }}$ \\
\hline E. citriodora & $32,83 \mathrm{a}$ & $-0,87 b$ & 63,14 & $0,95 * *$ & 0,0100 \\
\hline C. nardus & $4,66 \mathrm{~b}$ & $-0,17 \mathrm{a}$ & 10,14 & 0,75 & 0,0800 \\
\hline Citronelal & $32,07 \mathrm{a}$ & $-0,63 b$ & 70,25 & $0,95^{*}$ & 0,0130 \\
\hline Media & 22,66 & $-0,53$ & & & \\
\hline
\end{tabular}

Segundo Gomes et al. (2008), dentre estes dois gêneros, o Aspergillus sp. foi constatado apresentando maior incidência e com proliferação em todas as variedades de feijão-caupi avaliadas. De acordo com Marino et al. (2008) a ocorrência de Aspergillus sp. e Penicillium sp. em genótipos de Phaseolus vulgaris L. também pode causar danos na qualidade e na produtividade desta cultura. A incidência desses fungos de armazenamento pode causar a deterioração das sementes que se manifesta por meio de várias alterações químicas e fisiológicas, sendo a mais evidente e importante o decréscimo na germinação e no vigor (BORÉM et al., 2000). Foram também detectados fungos do gênero Aspergillus, Penicillium spp. e Fusarium spp. Segundo Mertz et al. (2007), Os dados discutidos acima mostram que os óleos essenciais são promissores para se chegar a novos produtos com atividade antifúngica de sementes de feijão armazenadas, os quais afetam de alguma maneira o desenvolvimento dos fungos Aspergillus sp., Curvularia sp., Fusarium sp., Nigrospora sp. e Penicillium sp., portanto propõem se estudar mais, a fim de tentar esclarecer o melhor modo de ação antifúngica desses óleos essenciais sobre esses fungos.

\section{CONCLUSÃO}

Os óleos essenciais de C. nardus, Eucalyptus citriodora e o composto citronelal tiveram bom potencial antifúngico, por reduzirem significativamente o número de colônias de Aspergillus sp., Curvularia sp., Fusarium sp., Nigrospora sp. e Penicillium sp. associados às sementes de feijão (Carioca, Caupi, Jalo e Preto), com destaque para o óleo de citronela e o composto citronelal.

\section{AGRADECIMENTOS}

À Universidade Federal do Tocantins, Campus de Gurupi por tornar possível a realização deste trabalho.

\section{REFERÊNCIAS BIBLIOGRÁFICAS}

AGUIAR, R. W. D.; OOTANI, M. A.; ASCENCIO, S. D.; FERREIRA, T. P. S.; DOS SANTOS, M. M.; DOS SANTOS, G. R. Fumigant Antifungal Activity of Corymbia citriodora and Cymbopogon nardus Essential Oils and Citronellal against Three Fungal Species. Scientific World Journal, p. 1-8, 2014.

AHMADI, F.; SADEGHI, S.; MODARRESI, M.; ABIRI, R.; MIKAELI, A. Chemical composition, in vitro antimicrobial, antifungal and antioxidant activities of the essential oil and methanolic extract of Hymenocrater longiflorus Benth., of Iran. Food and Chemical Toxicology, v. 48, n. 5, p. 1137-1144, 2010.

BORÉM, F. M.; SILVA, R. F.; HARA, T.; MACHADO, J. da C. Ocorrência de fungos no ar e em sementes de feijão (Phaseolus vulgaris L.) armazenadas em ambientes com equipamento modificador de atmosfera. Ciência Agrotécnica, v. 24, n. 3, p.195-202, 2000. 
CÂMARA, C. R. S.; URREA, C. A.; SCHLEGEL, V. Pinto Beans (Phaseolus vulgaris L.) as a Functional Food: Implications on Human Health. Agriculture, v. 3, n. 1, p. 90-111. 2013.

CASTRO, D. P.; CARDOSO, M. G.; MORAES, J. C.; SANTOS, N. M.; BALIZA, D. P. Não preferência de Spodoptera frugiperda (Lepidóptera: Noctuidae) por óleos essenciais de Achillea millefolium L. e Thymus vulgaris L., Revista Brasileira Plantas Medicinais, v. 8, n. 4, p. 27-32, 2006.

CHANG, H. T.; CHENG, Y. H.; WU, C. L.; CHANG, S. T.; CHANG, T. T.; SU, Y. C. Antifungal activity of essential oil and its constituents from Calocedrus macrolepis var. formosana Florin leaf against plant pathogenic fungi. Bioresource Technology, v. 99, n. 14, p. 6266-70, Sep 2008.

COX, S.D.; MANN, C.M.; MARKHAM, J.L.; BELL, H.C.; GUSTAFSON, J.E.; WARMINGTON, J.R.; WYLLIE, S.G., 2000. The mode of antimicrobial action of essential oil of Melaleuca alternifolia (tea tree oil). Journal Applied Microbiology. v. 88, n. 1, p. 170-175. 2000.

DAVID, E. F. S.; MISCHAN, M. M. BOARO, C. S. F. Desenvolvimento e rendimento de óleo essencial de menta (Mentha x piperita L.) cultivada em solução nutritiva com diferentes níveis de fósforo. Revista Biotemas, v.20, n. 2, p. 15-26, 2007

FERREIRA, D. F. Programa de análises estatísticas (statistical analysis sotware) e planejamento de experimentos - SISVAR 5.0 (Build 67). Lavras: DEX/UFLA, 2003.

GOMES, D. P.; SILVA, G. C.; KRONKA, A. Z.; TORRES, S. B.; SOUZA, J. R. Qualidade fisiológica e incidência de fungos em sementes de feijão-caupi produzidas do Estado do Ceará. Revista Caatinga, v.21, n. 2, p. 165-171, 2008.

HARUNA, I.M.; USMAN, A. Agronomic efficiency of cowpea varieties (Vigna unguiculata L. Walp) under varying phosphorus rates in Lafia, Nasarawa State, Nigeria. Asian Journal of Crop Science, v.5, n. 2, p. 209-215, 2013.

KHALEDI, N.; TAHERI, P.; TARIGHI, S. Antifungal activity of various essential oils against Rhizoctonia solani and Macrophomina phaseolina as major bean pathogens. Journal of Applied Microbiology, v. 118, n. 3, p. 704-17, Mar 2015.

LUCCA FILHO, O.A. Testes de sanidade de sementes de milho. In: SOAVE, J.; Wetzel, M.M.V. da S. Patologia de sementes. Campinas: Fundação Cargill/ ABRATESCOPASEM, 1987, p. 430-440.

MACHADO, J. C. Padrões de tolerância de patógenos associados a sementes. Revisão Anual de Patologia de Plantas, v. 2, p. 229-262, 1994.

MARCO, C. A.; INNECCO, R.; MATTOS, S. H.; BORGES, N. S. S.; NAGA, E. O. Características do óleo essencial de capim-citronela em função de espaçamento, altura e época de corte. Horticultura Brasileira, v.25, p. 42932, 2007.

MARINO, R. H.; MESQUITA, J. B.; ANDRADE, K. V. S. DE; COSTA, N. A. DA; AMARAL, L. A. Incidência de fungos em sementes de Phaseolus vulgaris L. provenientes do Estado de Sergipe. Revista Brasileira de Ciências Agrárias, v.3, n. 1 p. 26-30, 2008.

MERTZ, L. M.; HENNING, F. A.; MAIA, M. de S.; MENEGHELLO, G. E.; HENRIQUES, A.; MADAIL, R. Qualidade fisiológica e sanitária de sementes de feijãomiúdo beneficiadas em mesa gravitacional. Revista Brasileira de Sementes, v. 29, n. 3, p. 01-08, 2007.

MOLEYAR, M.; NARASIMHAM, P. Antifungal activity of some essential oil components, Food Microbiology, v.3, n. 4, p. 331-336, 2004.

MONTEMOR, C. L. B.; CASA, R. T.; OLIVEIRA, F.S.; KUHNEM JUNIOR, P. R.; BOGO, A.; CORRÊA, T. R. Detecção de Colletotrichum lindemuthianum em sementes do banco de germoplasma de feijão da Universidade do Estado de Santa Catarina. Revista de Ciências Agroveterinárias, v.11, n. 1, p. 48-53, 2011.

NOVEMBRE, A. D. L. C.; FILHO, J. M. Tratamento fungicida e conservação de sementes de feijão. Revista Brasileira de Sementes, v.13, n. 2, p. 105-113, 1991.

PAWAR, V.C.; V.S. THAKER. In vitro efficacy of 75 essential oils against Aspergillus niger. Mycosis, v.49, n. 4, p. $316-323,2006$.

RANI, P.R.; CHELLADURAI, V.; JAYAS, D.S.; WHITE, N.D.G.; KAVITHA-ABIRAMI, C.V. Storage studies on pinto beans under different moisture contents and temperature regimes. Journal of Stored Products Research, v.52, p. 78-85, 2013.

REY, M.S.; LIMA, N.B.; SANTOS, J.; PIEROBOM, C.R. Transmissão semente-plântula de Colletotrichum Lindemuthinum em feijão (Phaseolus vulgaris). Arquivos do Instituto Biológico, São Paulo, v.76, n.3, p. 465-470, 2009.

RODRIGUES, A. A. C; MENEZES, M. Detecção de fungos endofíticos em sementes de caupi provenientes de Serra Talhada e de Caruaru, Estado de Pernambuco. Fitopatologia Brasileira, v.27, n. 5, p. 532-537, 2002.

SALLIS, M. da G. V.; LUCCA-FILHO, O. A.; MAIA, M. de $\mathrm{S}$. Fungos associados às sementes de feijão-miúdo (Vigna unguiculata (L.) Walp.) produzidas no Município de São José do Norte (RS). Revista Brasileira de Sementes, v. 23, n. 1, p. 36-39, 2001.

SANTOS, S.C.; FERREIRA, F.S.; ROSSI-ALVA, J.C.; FERNANDEZ, L.G. Atividade antimicrobiana in vitro do extrato de Abarema cochiliocarpos (Gomes) Barnaby \& Grimes. Revista Brasileira de Farmacognosia, v.17, n. 2, p. 215-219, 2007 
SCHWAN-ESTRADA, K. R. F.; STANGARLIN, J. R. Extratos e óleos essenciais de plantas medicinais na indução de resistência. In: CAVALCANTI, L S.; DI PIERO, R. M.; CIA, P.; PASCHOLATI, S. F.; RESENDE, M. L. V.; ROMEIRO, R. S. (Ed.). Indução de resistência em plantas a patógenos e insetos. Piracicaba: FEALQ, c.5, p. 125-138, 2005.

SILVA, D.M.M.H.; BASTOS, C.N. Atividade Antifúngica de Óleos Essenciais de Espécies de Piper Sobre Crinipellis perniciosa, Phytophthora palmivora e Phytophthora capsici. Fitopatologia Brasileira, v.32, n. 2, p. 143-145, 2007.

SIMIC, A.; RANCIC, A.; OKOVIC, M. D.; RISTIC, M.; GRUJIC-JOVANOVIC, S.; VUKOJEVIC, J.; MARIN, P. D. Essential Oil Composition of Cymbopogon winterianus and Carum carvi and Their Antimicrobial Activities. Pharmaceutical Biology, v.46, n. 6, p. 437-441, 2008.

SOUZA, D.C.; LOURENÇO, S.A.; BASSETTO, E.; AMORIM, L. Progresso temporal da podridão parda do pessegueiro em áreas não tratadas e tratadas com fungicidas. (Resumo) Summary Phytopathologica, v.32 (suplemento), p.32, 2005.

SOYLU, E.M, SOYLU, S, KURT, S. Antimicrobial activities of the essential oils of various plants against tomato late blight disease agent Phytopthora infestans. Mycopathologia, v.161, n. 2, p. 119-28, 2006.

STONE, L.F.; FERREIRA, E.P.B.; DIDONET, A.D.; HEINEMANN, A.B.; OLIVEIRA, J.P. Correlação entre a produtividade do feijoeiro no sistema de produção orgânica e atributos do solo. Revista Brasileira de Engenharia Agrícola e Ambiental, v.17, n. 1, p. 9-25, 2013.

SU, Y.C.; HO, C.I.; WANG, I. C.; CHANG, S. T. Antifungal activities and chemical compositions of essential oils from leaves of four eucalypts. Taiwan Journal of Forest Science, v.21, n. 1, p. 49-61. 2006.

TORRES, S.B.; PEIXOTO, A.R.; CARVALHO, I.M.S. Qualidade Sanitária e fisiológica de sementes de feijãomacássar (Vigna unguiculata (L.) Walp.) da região do submédio São Francisco. In: Resumos, Congresso Brasileiro de Sementes, 10, Curitiba, PR. p. 129, 1997.

VANDENDOLL, H.; KRATZ, P.D.J.A. Generalization of the retention index system including linear temperature programmed gas-liquid partition chromatography. Journal Chromatography, v. 11, p. 463-471, 1963.

VELLUTI, A.; MARIN, S.; GONZALEZ, P.; RAMOS, A. J.; SANCHIS, V. Initial screening for inhibitory activity of essential oils on growth of Fusarium verticillioides, $F$. proliferatum and $F$. graminearum on maize-based agar media. Food Microbiology, v. 21, n. 6, p. 649-656, 2004.

VILELA，G.R.; ALMEIDA，G.S.; DARCE，M.A.B.R.; MORAES, M.H.D.; BRITO, J.O., SILVA, M.F.G.F.; SILVA, S.C. ; PIEDADE, S.M.S.; CALORIDOMINGUES, M.A.; GLORIA, E.M. Activity of essential oil its major compounds 1,8 - cineole from Eucalyptus globules, against the storage fungi Aspergillus avus and Aspergillus parasiticus. Journal of Stored Products Research, v.45, n. 2, p. 108-111, 2009.

ZOTTICH, U.; CUNHA, M.; CARVALHO, A.O.; DIAS, G.B.; CASARIN, N.; VASCONCELOS, I.M.; GOMES, V.M. An antifungal peptide from Coffea canephora seeds with sequence homology to glycine-rich proteins exerts membrane permeabilization and nuclear localization in fungi. Biochimica et Biophysica Acta (BBA) - General Subjects, v.1830, n. 6, p. 3509-3516, 2013. 